\title{
Misión de la Medicina Interna y su influencia en el conjunto del hospital
}

De Portugal Álvarez. J. Misión de la Medicina Interna y su influencia en el conjunto del hospital. An Med Interna (Madrid) 2005; 22: 559-560.

La Medicina Interna supone la atención integral al hombre enfermo mediante el método clínico y el saber científico, en el ámbito de toda la patología médica o partes de la patología médica. El primer caso, cuando la Medicina Interna se dedica a toda la patología médica se refiere al internista generalista, el internista por antonomasia. El segundo, la dedicación de la Medicina Interna a una parte de la patología médica ha supuesto la evolución del modelo anterior a dos versiones parecidas pero no iguales: una, la del internista subespecializado en una parte de la patología médica, pero que mantiene su actividad generalista, la otra, la del especialista exclusivo en una de esas partes pero que mantiene la formación y mentalidad internistas. Estos tres tipos de internitas, con mayor cualificación como tales, desde el primero al tercero, son con el especialista en sentido estricto, dedicado exclusivamente a una parte de la patología médica, sin formación y mentalidad internistas y, por tanto, fuera del ámbito de la Medicina Interna, los profesionales que se mueven en el campo de la patología médica.

La aparición y desarrollo de las subespecialidades médicas han tenido unas causas razonables y han contribuido al progreso clínico y científico de la medicina, pero también han introducido problemas en la identificación y el espacio de la medicina interna, que por conocidos y en buena medida sobrepasados, no es oportuno tratar aquí. Pero sí me voy a referir a un viejo problema que empezamos a sentir los internitas con la aparición de las subespecialidades médicas y que se ha ido agravando progresivamente. Tal problema se enmarca en la disyuntiva extensión-profundidad de conocimientos y habilidades. El internista se caracteriza por un predominio de la extensión en detrimento de la profundidad, mientras que en el caso del especialista es al revés. Esta distinción esquemática ha adquirido categoría de tópico, pero conviene decir que el rasgo positivo del internista, la mayor extensión de conocimientos y habilidades no es puramente cuantitativa sino que está cualificada por el sentido integrador, sintetizador y unificador. Pero en cualquier caso hay algo que los internistas debemos reconocer: nuestra baja competitividad en el manejo de enfermedades médicas en relación con el especialista correspondiente. Esta cuestión puede ser explicada y discutida, pero se reconoce, abierta o tácitamente, tanto en el ámbito de los médicos como de los pacientes.
Sin embargo, esta supuesta baja competitividad del internista no parece contemplarse en el plano de la medicina hospitalaria, toda vez que pacientes con una misma enfermedad, ingresan indistintamente en áreas de Medicina Interna o de especialidad. Los pacientes con patología médica que ingresan en el hospital y su posible distribución, son los siguientes:

1. Pacientes críticos, que derivan a unidades especiales de cuidados críticos.

2. Pacientes agudizados, con procesos ya diagnosticados y tratados, que van o deben ir a unidades de corta estancia.

3. Pacientes con procesos agudos "de novo", que según los casos son dirigidos a la unidad de corta estancia o a servicios de subespecialidad o de Medicina Interna.

4. Pacientes no agudos, que ingresan en Medicina Interna o la subespecialidad más próxima a su cuadro clínico.

Estos pacientes agudos o no agudos que ingresan indistintamente en Medicina Interna y especialidades, son fundamentalmente de tres tipos:

-Pacientes con claros requerimientos de atención especializada, es decir, con patología especial, bien definida, de una parte de la patología médica que demanda el manejo de un equipo especializado. Estos pacientes son dirigidos a la subespecialidad correspondiente.

-Pacientes comunes, sin requerimientos de atención especializada, es decir, con enfermedad de una parte de la patología médica, pero enfermedad de manifestación común o habitual que no requiere necesariamente la atención del especialista. Estos pacientes son distribuidos unas veces aleatoriamente, otras con criterios variables e imprecisos, a la subespecialidad correspondiente o a Medicina Interna.

-Finalmente, una masa heterogénea de pacientes inclasificables, crónicos, seniles, terminales, con abundante pluripatología, que se vierten monótonamente en el Servicio de Medicina Interna.

El segundo tipo de pacientes, aquellos con patología manifiesta de un órgano o sistema de expresión común, sin especiales compromisos diagnósticos y terapéuticos y con frecuente pluripatología asociada o derivada, es el tipo más frecuente que ingresa en el hospital. Y como queda dicho unas veces se deriva, según las manifestaciones clínicas más aparentes, a la especiali- 
dad y otras a Medicina Interna. Pero este tipo de paciente debería ingresarse preferentemente en Medicina Interna, mientras que el paciente con condiciones o circunstancia especiales en su patología y que necesita atención diagnóstica y/o terapéutica requieren su atención adecuada y pertinente. El segundo es el médico que por definición convencional se dedica a una parte anatomofisiológica del organismo, al margen de la cualidad de su patología. Este tipo de paciente común que venimos refiriendo cae habitualmente en el área de especialidad dirigido más con un criterio parcialista que especialista y pensamos que por el contrario estos pacientes deberían ingresarse preferentemente en Medicina Interna, mientras que el paciente con patología cualificada que necesita atención diagnóstica y/o terapéutica especializada, sería el adecuado para el área de especialización.

Ésta poco racional distribución de pacientes en la medicina del hospital, sedimentada por la inercia del uso, no parece beneficiosa para los intereses de la Medicina Interna ni para los de las especialidades. La primera porque se ve expoliada de muchos pacientes que por capacitación podría atender y las segundas porque se saturan de pacientes de su parte anatomofisiológica, con patología común y sin necesidad de atención especializada, con lo cual se desfigura su verdadero papel de especialidades y se bloquean otras tareas asistenciales y de investigación.

Sin embargo, ésta poco pertinente distribución de pacientes en el área de Medicina de Hospital, es aceptada satisfactoriamente por los gestores hospitalarios. Cuando nuestros gestores sanitarios y mucho antes los de la medicina americana han comenzado a utilizar métodos modernos de análisis con sistemas de registro informatizados, han comprobado la rentabilidad de los Servicios de Medicina Interna que atienden la mayor parte de los pacientes del área de medicina y, en general, con índices cualitativos semejantes o mejores que los de las subespecialidades. Por ejemplo, comparando estancias medias en Medicina Interna y en subespecialidades de los principales GRD ingresados en 1999 en nuestro hospital, se observa que, en general, son más altas en las subespecialidades. En una muestra analizada hace algunos años en el Hospital Clínico de Salamanca, se pone de manifiesto que las estancias medias de cuatro GRD eran más bajas cuando eran manejados en Medicina Interna que en la subespecialidad correspondiente y también en la primera eran menores el número de pruebas solicitas al paciente y el número de interconsultas que en las segundas. Estas dos últimas circunstancias que son, en alguna manera inherentes al modo de hacer del internista, influyen necesariamente en la estancia media.

Pero si ésta, más o menos, aleatoria distribución de pacientes puede resultar beneficiosa para la dinámica y el gasto hospitalarios, insisto que no lo es para lo que deben ser la Medicina Interna y las subespecialidades. Sería interesante establecer unos criterios consensuados (ya realizados en algunos hospitales americanos) entre Medicina Interna y las distintas subespecialidades, en todas y cada una (al menos en las de mayor prevalencia) de las enfermedades de la patología médica, para racionalizar su distribución. Esto, sin duda, elevaría la pertinencia de la actividad clínica, potenciaría las verdaderas funciones de la subespecialidad y evitaría o disminuiría la pérdida de pacientes reagudizados y determinados agudos, a unidades de corta estancia.

Por otra parte, pero en el sentido de lo anterior, sería necesario elevar la competitividad del internista en relación con la del subespecialista en los procesos más frecuentes de la práctica médica, profundizando en su conocimiento científico y adqui- riendo habilidades en algunas técnicas clínicas: ecocardiografía, endoscopias, pruebas funcionales, tomas biópsicas, etc. el internista podría seguir siendo generalista, pero con semejante competencia clínica en determinadas enfermedades de presentación común, que el subespecialista correspondiente.

Probablemente las consideraciones anteriores tengan una validez transitoria y pierdan interés en un futuro no muy lejano. La medicina cambia como cambia la sociedad, inducida por factores sociales, políticos, económicos, culturales, etc. algunos de estos cambios son predecibles y otros incluso ya se ha iniciado. Recuérdese los producidos por el envejecimiento de la población, la tecnología médica, la cibermedicina, la política de gestión, la potenciación de la medicina primaria, etc., pero me voy a referir aquí a tres tipos de circunstancias que van a generar importantes cambios en el fundamento y la práctica de la medicina.

1. La prevalencia de algunas, quizá muchas, enfermedades es previsible que descienda notablemente como consecuencia de programas eficaces de prevención, tratamientos altamente resolutivos, manipulaciones genéticas, etc., y esto reducirá necesariamente el contenido de algunas subespecialidades, que a pesar del aporte, nunca compensatorio, de eventuales enfermedades emergentes y reemergentes, verán comprometida su viabilidad.

2. Nuestro conocimiento de la fisiopatología de las enfermedades es cada vez más profundo. Estamos llegando al conocimiento de mecanismos etiopatogénicos más esenciales, más elementales, más primarios. Recuérdese al respecto lo que al conocimiento y al manejo de muchas enfermedades, han introducido, o están introduciendo, la inmunología, la enzimología, la biología molecular, la ingeniería genética, la trasmisión química, la manipulación de receptores y otras muchas adquisiciones de la ciencia médica que van a hacer que se desborde la ordenación nosológica clásica de las enfermedades y que su organización basada en órganos y sistemas quede sobrepasada. Es posible que ello conduzca a la creación de nuevas áreas de la Medicina, constituidas a nivel más elemental y básico que el de las actuales especialidades organobasadas. Estas nuevas áreas alojarán problemas comunes a varias de las subespecialidades tradicionales y el subespecialista concreto de corte actual verá comprometida su existencia como tal.

3. El hospital futuro, establecido sobre la actual transferencia del control médico al de la administración-empresa, modificará el criterio de hospitalización de pacientes impelido por el enorme gasto que genera.

Estos hospitales serán principalmente cenros de atención a pacientes agudos, graves y situaciones críticas que requieran alto grado de tecnología, con áreas o unidades seguramente más polivalentes que específicas. En estos hospitales, los dos tipos de médicos operativamente más importantes serán el técnico y el "hospitalista", médico de formación generalista y de actividad polivalente, será el encargado clínico de todos los pacientes ingresados, agudos y no agudos, con el concurso ocasional del especialista en situaciones concretas. El papel asistencial del "hospitalista", cuyo antepasado más cercano es el internista generalista, está creciendo significativamente en hospitales europeos y americanos.

\section{J. DE PORTUGAL ÁLVAREZ}

Servicio de Medicina Interna II. Hospital General Universitario Gregorio Marañón. Madrid 

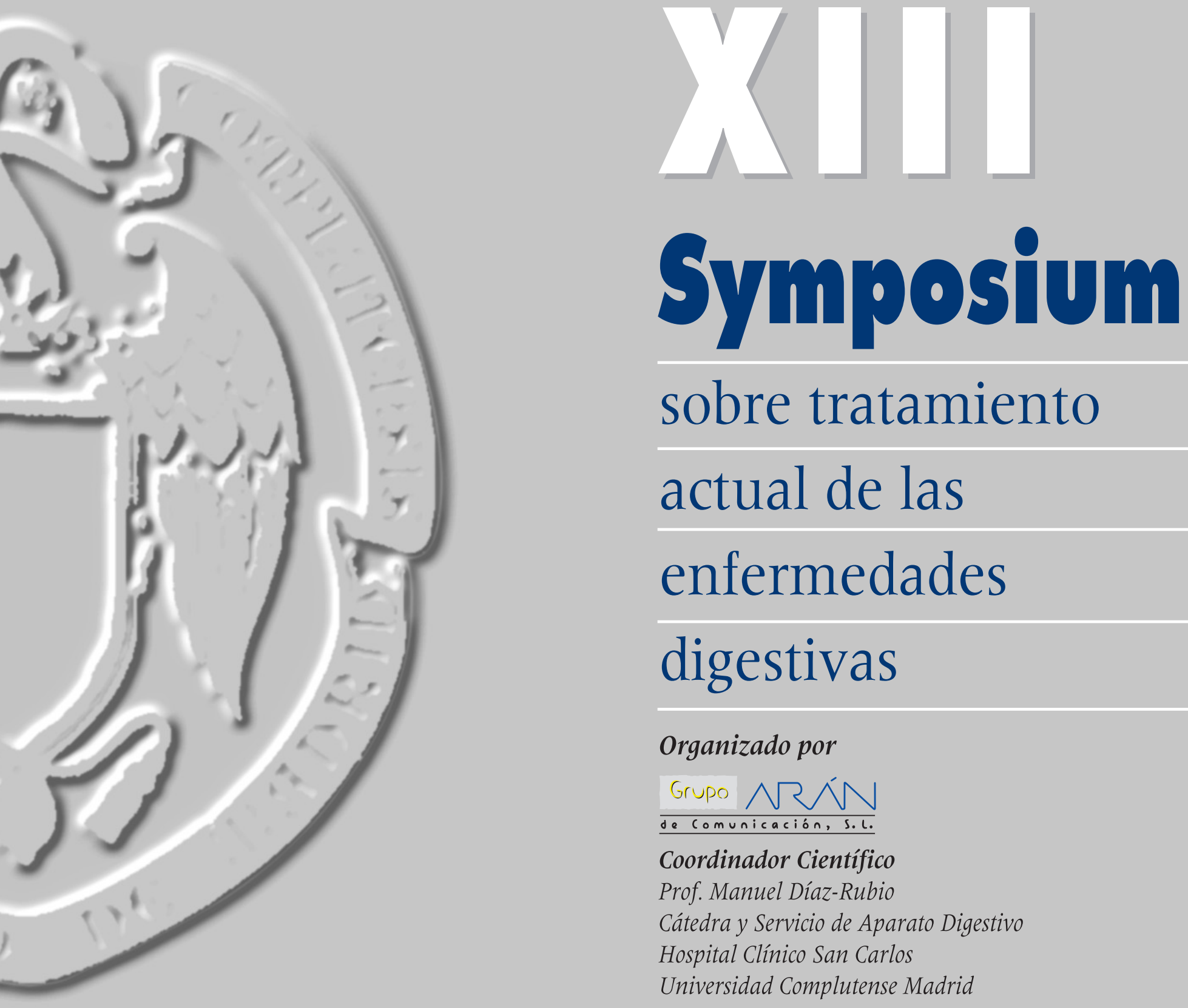

\section{sobre tratamiento}

\section{actual de las}

\section{enfermedades}

\section{digestivas}

\section{Organizado por}

$\frac{\text { Grupo de comunicación, s.l. }}{\text { dén }}$

Coordinador Científico

Prof. Manuel Díaz-Rubio

Cátedra y Servicio de Aparato Digestivo

Hospital Clínico San Carlos

Universidad Complutense Madrid

\section{www.grupoaran.com/enfermedades2006}

Madrid, 2 - 3 de marko de 2006 


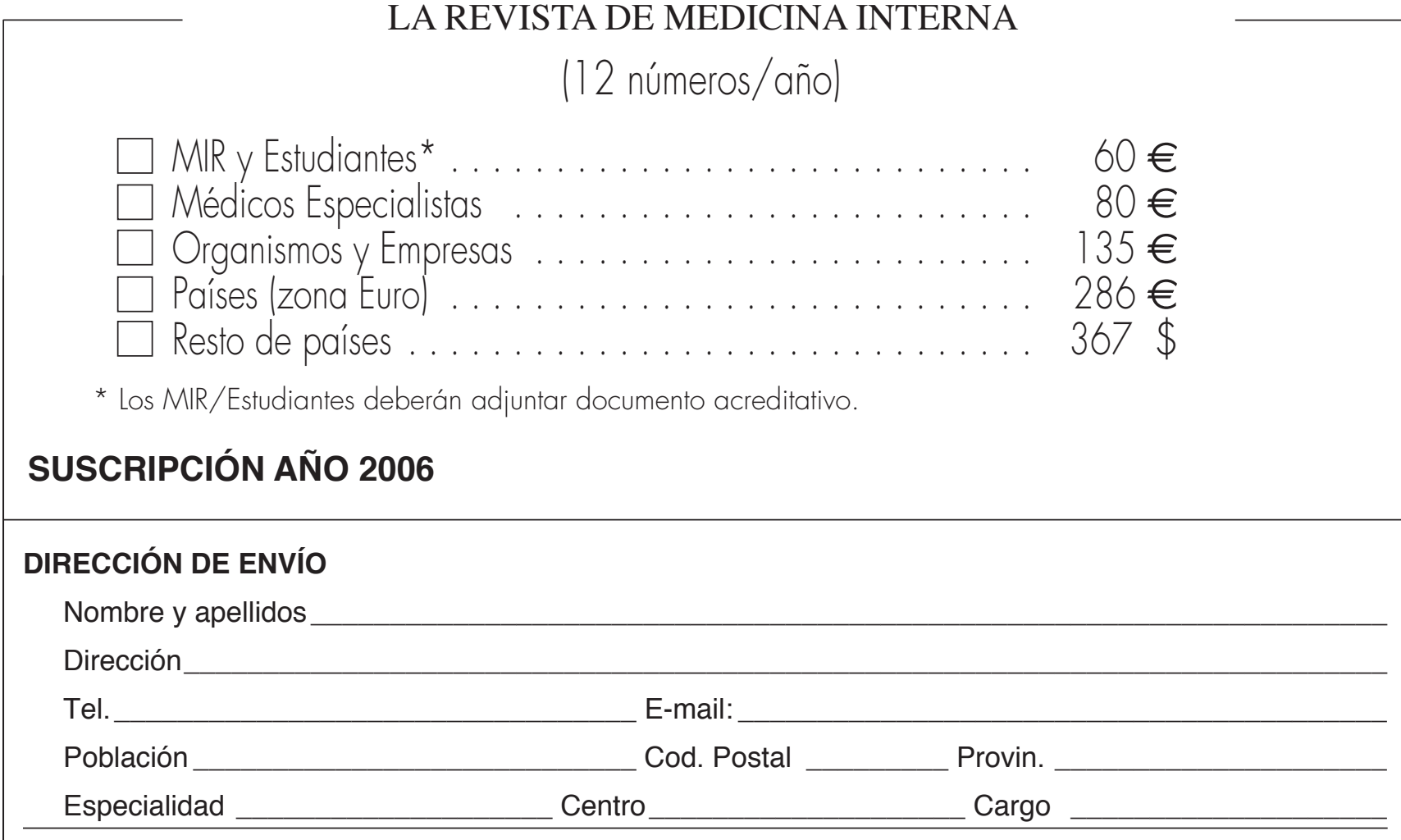

\section{SUSCRÍBANME A:}

$\square$ A través de mi cuenta bancaria (cumplimento autorización adjunta)

$\square$ Mediante talón $\mathrm{n}$. $^{\circ}$ que adjunto

ANALES DE

MEDICINA

INTERNA

$\square$ Contra reembolso

(12 números/año)

ORDEN DE PAGO POR DOMICILIACIÓN BANCARIA

ANALES DE MEDICINA INTERNA

BANCO/CAJA

DIRECCIÓN POBLACIÓN C.P.

TITULAR DE LA CUENTA

CÓDIGO C/C.: BANCO

SUCURSAL

D.C.

N. ${ }^{\circ}$ CUENTA

Ruego a ustedes se sirvan tomar nota de que hasta nuevo aviso, deberán adeudar en mi cuenta con esa entidad el recibo o letra que anualmente y a mi nombre les sean presentados para su cobro por

Les saluda atentamente,

(Firma)

de

de 20

\section{Más información o envíos a:}

